\title{
Present situation and progress of pancreatic cancer radiotherapy
}

Gao Yu', Yan Wenming ${ }^{2}$ (Corresponding author)

Department of Radiotherapy of The Affiliated Hospital of Inner Mongolia Medical University Hohhot Inner Mongolia 010050

Abstract: Purpose: The research purpose is to effectively improve treatment effect of postoperative pancreatic cancer. Approaches: Adjuvant radiotherapy and chemotherapy; Palliative radiotherapy is adpoted for patients whose pancreatic cancer cannot be removed through excision. Comprehensive treatment approach is adopted where gemcitabine is added during radiotherapy. Result: The treatment effect of palliative operation is a little bit better than the traditional operative treatment, adoption of comprehensive treatment is obviously superior to the aforesaid two treatment approaches. Conculsion: Comprehensive treatment effectively improves survival rate of patients, and the survival time is greatly extended.

Operation is generally adopted for pancreatic cancer treatment, but the effect is unsatisfactory. The average lifetime of patients after palliative operation treatment is only 5 to 76 months, and the survival rate after five years only reaches $8 \%$ to $15 \%$, while the curative effect of radical resection is a little bit better, but the survival rate after five years also only reaches $12 \%$ to $24 \%$, and the average lifetime is about 10 to 19 months. In recent years, with gradual progress of medical treatment level, clinical cases of adopting radiotherapy for pancreatic cancer are increasing, but its main effect is palliative or auxiliary. This article refers to plenty of documentation, makes thorough study of present situation and progress of pancreatic cancer radiotherapy, put forward its own suggestions and opinions, and have certain reference value.

Keywords: pancreatic cancer; radiotherapy; present situation; progress

Incidence of pancreatic cancer is comparatively low (about one ten-thousandth), but this disease has high fatality rate and rapid development speed, and is an extremely dangerous malignant tumor (Survival rate of the patients after one year is only $8 \%$, and it is only $3 \%$ after five years). Operative treatment is traditional treatment method for pancreatic cancer, but but the effect is unsatisfactory, while treatment effect of palliative operation is obviously improved (Survival rate after five years can reach $8 \%$ to $15 \%$ ). With improvement of medical treatment level, clinical cases of adopting radiotherapy for pancreatic cancer are increasing, which provides basis for further research of pancreatic cancer treatment.

\section{Preoperative radio therapy of pancreatic cancer}

\subsection{The advantages of preoperative radiotherapy}

Comparing with the postoperative chemotherapy, radiotherapy and chemotherapy, preoperative radiotherapy and chemotherapy can significantly improve the resection rate, can reduce tumor spread, those spreads are caused by the operation, which are sensitive to radiotherapy, and it can better oxygenate with tumor cells. According to the few documentation and reports, the local recurrence rate is much lower than that of postoperative radiotherapy and chemotherapy 


\subsection{Palliative radiotherapy for pancreatic cancer that cannot be resected}

Patients with pancreatic cancer also has such symptoms as gastrointestinal tract obstruction, losing weight, nausea and vomit, inappetence, obstructive jaundice and ache, which bring overwhelming pain to them, meanwhile, it also leads to significant reductions of life quality, severe impact and final death of patients. Therefore, radiotherapy should be cautiously applied, especially for those patients with bad physical situation and extensive lesions. For those incurably ill patients that local resection cannot by done, palliative radiotherapy should be used to relieve their symptoms.

Locally advanced pancreatic cancer that cannot be resected has bad prognosis. For comment on curative effect of this kind of patients, survival situation, local control rate, life quality improvement rate and symptom relief rate of patients should be comprehensively considered. In reports of Andre and others, during the process of clinical treatment of locally advanced pancreatic cancer, radiotherapy, injection of 5-FU and cis-platinum are applied, regarding to behavior status, $30 \%$ of them are deteriorated, $47 \%$ are stable, and $23 \%$ are improved; regarding to weight: $3.5 \%$ of them gain weight, $7 \%$ are stable, $89.5 \%$ lose weight; Pain: $65 \%$ of them totally lose pain, $9 \%$ are relieved, $22 \%$ are stable, and $4 \%$ are deteriorated. JIA Zhengeng and others in China reported that pain relief rate after radiotherapy of 45 cases of pancreatic cancer operation is $95.5 \%$ (43/45), including 55.5\% (25/45) of total disappearing of pain. WU Zheng and others reported that the total effective rate reached $76.1 \%$ after extracorporeal radiotherapy of 21 cases of pancreatic cancer, hereinto: status of nine patients was obviously relieved, the pain of seven patients was totally relieved. We can see from these materials that radiotherapy can effectively relieve the pain of patients for those with locally advanced pancreatic cancer.

\section{Postoperative radiotherapy of pancreatic cancer}

Generally speaking, the approach that can effectively cure pancreatic cancer is still radical resection operation, but as many major organs are around the pancreas, for example, spinal cord, kidney, liver, small intestine, duodenum and stomach restrict eradication of the operation.

In order to further specify curative effect of auxiliary radiotherapy and chemotherapy after pancreatic cancer radical surgical resection, RTOG of the U.S. started one random grouping research (RTOG-97-04) in August 1998 to compare curative effect of application of 5-FU (group one) or gemcitabine (group two) before or after radiotherapy and chemotherapy after pancreatic cancer radical surgical resection. The detailed scheme is: radiotherapy and chemotherapy begin within three to six weeks after the operation. The first group uses $250 \mathrm{mg} / \mathrm{m} 2$ of 5-FU every day for total three successive weeks; The second group uses $1000 \mathrm{mg} / \mathrm{m} 2$ of gemcitabine every day, once a week, total three weeks. Radiotherapy and chemotherapy begin within one to two weeks after the aforesaid chemotherapy, meanwhile, 5 -FU continues to be instilled. The radiotherapy scheme is $1.8 \mathrm{~Gy}$ once every day, and the added dosage begins to be changed till total dosage $50.1 \mathrm{~Gy} / 5.5$ weeks after $15 \mathrm{~Gy}$. Scheme of the first group is: the time rang of one period is rest for two weeks and successive application for four weeks, total six weeks, $250 \mathrm{mg} /(\mathrm{m} 2 . \mathrm{d})$ of 5 -FU keeps to be instilled for total two periods. Scheme of second group is: the time rang of one period is rest for one week and successive application for two weeks, total three weeks, $1000 \mathrm{mg} /(\mathrm{m} 2 . \mathrm{d})$ of gemcitabine is instilled once a week for total three periods.

\section{Radiotherapy and chemotherapy for postoperative pancreatic cancer}

In recent years, gemcitabine is paid much attention during treatment of pancreatic cancer. Its main therapeutic scheme is as follows: 


\subsection{Radiotherapy adopts standard therapeutic scheme}

1.8 Gy once a day, five times a week, and the total dosage is $50.4 \mathrm{~Gy}$; the dosage of gemcitabine is lower than the standard dosage. According to the reports including that of Poggi, during one period of clinical research of comprehensive treatment of gemcitabine and radiotherapy for pancreatic cancer which cannot be resected, the aforesaid scheme is adopted for radiotherapy. Gemcitabine is used once a week for total five times. The dosage starts from 350 $\mathrm{mg} / \mathrm{m}^{2}$, and is gradually increased, the final maximum tolerance dose is $440 \mathrm{mg} / \mathrm{m}^{2}$. During two periods of clinical research of GALGB, therapeutic scheme of gemcitabine is twice a week (Medication is on Monday and Thursday), and the appropriate dosage is $40 \mathrm{mg} / \mathrm{m}^{2}$.

\subsection{Standard dosage of gemcitabine is applied}

1.8 Gy once a day for total ten times of two successive weeks. After one week of rest, the aforesaid chemotherapy and radiotherapy scheme are repeated. The total radiotherapy dosage is $50.4 \mathrm{~Gy}$, which is the same with the common standard dosage, but is completed by three phases.

\section{Other therapeutic techniques of pancreatic cancer}

\subsection{D-CRT or IMRT}

Domestic and foreign scholars are making clinical research of 3D-CRT or IMRT of pancreatic cancer. Currently, there is no mature curative scheme. Seen from the existing limited materials, 3D-CRT or IMRT of pancreatic cancer has the following features comparing with the conventional radiotherapy: (1) The target range is narrowed; (2) Many scholars adopts unconventional partitioning method for irradiation. The total course of treatment is shortened with fewer irradiation times and larger dosage of every time. Seen from limited clinical reports, short-term curative effect of this technique is comparatively good, which can obviously reduce radiotherapy reaction and improve tolerance of the patients. Scholars including Xu Weidong reported that they used three-dimensional stereotactic radiotherapy for 15 cases of pancreatic cancer that cannot be treated by surgery, and one-year survival rate reached $73.3 \%$, two-year survival rate reached 33.3\%. Scholars including Yu Jinming reported that they used stereotactic radiotherapy for 13 cases of pancreatic cancer that cannot be treated by surgery, and one-year survival rate and two-year survival rate were respectively $92.3 \%$ and $70 \%$.

3D-CRT or IMRT of pancreatic cancer is still in the preliminary phase, demarcation of target volume, best partitioning method, total dosage and other issues still need to be further specified in the clinical research in the future and are worth further research.

\subsection{Combination of tomotherapy and radiotherapy}

Comparing with traditional curative method, the biggest feature of Tomotheraopy of pancreatic cancer is higher conformation number of tumor dose and more precise of intensity adjustment of tumor dose, and finer dose adjustment of normal tissues around the tumor, especially for such tumors with "complex position" as pancreatic cancer, advantages of Tomotherapy are more obvious, and Tomotherapy can distinctly reduce occurrence rate of adverse reaction of the tumor

\subsection{Combination of particle implantation and radiotherapy}

Pancreatic cancer is hypoxic tumor, has strong resistance to radioactive rays, and extremely sensistive to Gamma ray. A small quantity of Gamma ray can destroy DNA. Radioactive particle $\mathrm{I}^{125}$ keeps emitting Gamma ray to prevent breeding of tumor cells and has small damage to the surrounding normal tissues. ${ }^{125}$ particle implantation to cure pancreatic cancer cannot only significantly improve life quality of the patients and increase local control rate of the tumor, but also 
is expected to improve long-term survival rate, and it is a prospective treatment method for pancreatic cancer treatment.

$\mathrm{I}^{125}$ particle implantation is implementation among pamernant radioactive source tissues, CT, MRI or ultrasound can work as imaging tool to guide particle implementation. Particle implementation under guidance of imaging can completely meet outline of the tumor, get accurate conformation number, and the rays completely cover the target section. The target section of the tumor can get the dose for radical curement of the tumor, and injectivity of the surrounding normal tissues is very low, which will not cause severve acute or later period damage.

\section{Conclusion}

This article makes research on application of radiotherapy technique in treatment of pancreatic cancer based on traditional treatment method of pancreatic cancer. Local control rate can be effectively increased through introduction of new radiotherapy technique, and the effect on extension of life time of hte patients is obvious. As radiotherapy has strong toxicity, the new adjuvant treatment method can reduce excision rate, and improve survival rate. With introduction of mor new techniques, radiotherapy will have better effect on treatment of pancreatic cancer.

\section{References}

1. Zong C and Zhang J, 2014, Radiotherapy progress of pancreatic cancer.PJC, vol. 2014(2): 242-244.

2. Qi Z and $\mathrm{Yu} Z, 2014$, Current situation of comprehensive treatment on pancreatic cancer during local advanced phase. Journal of Diseases Monitor \& Control, vol. 06(6): 345-347.

3. Qi C, Lauren MR, Joseph MH, 2015, Research progress of stereotactic radiotherapy in pancreatic cancer. Chin J Cancer Res, vol. 2015(4): 349-357.

4. Cheng D, Li H, Zhao S, et al., 2016, Pancreatic cancer in operation of mobile linear accelarator during radiotherapy advanced phase. Chinese Journal of Hepatobiliary Surgery, vol. 22(3): 155-158.

5. Wang Y and Li Y, 2015, Current status and progress of irradiating treatment on pancreatic cancer in radionuclide. Chinese Journal of General Surgery, vol. 24(3): 408-412.

6. Ren G and Xia T, 2016, Research progress of changes of radiotherapy dose mode for pancreatice cancer. Chinese Journal of Colorectal Disease (Electronic Edition), vol. 5(2): 121-126.

7. Nilupi A and Zhang H, 2017, Current situation of internal medicine treatment on advanced pancreatic cancer. Oncology Progress, vol. 15(4): 371-376. 